\title{
Physiotherapy following cardiac surgery: Program comparison
}

\author{
D. Pulmane ${ }^{1,2}$, A. Vetra ${ }^{1}$, R. Lacis ${ }^{2}$, and D. Driba ${ }^{1,2}$ \\ ${ }^{1}$ Rīga Stradiņš University, Riga, Latvia \\ ${ }^{2}$ Stradinšs Clinical University Hospital, Latvia
}

\begin{abstract}
The objective is to examine and compare the usability of two physiotherapy programmes, analyzing respiratory function in patients before and after cardiac surgery in hospital during seven postoperative days (POD).

Quantitative randomized prospective study of 157 patients before and after the valve replacement surgery, coronary artery bypass graft and combined surgeries, who moved independently. Participants were randomized into two groups (1 and 2$)$ with different physiotherapy programmes. The routine physiotherapy of breath-enhancing techniques, micro-circulation improvement were used for the first group, for the second group modified physiotherapy - the improvement of inspiration muscles, mm. quadriceps. gluteus max strength. Anthropometric measurements were defined for both groups on the day before surgery and during spirography dynamic indicators - forced vital capacity (FVC), forced expiratory volume in the $2^{\text {nd }}$ second (FEV1), Tiffeneau index (FEV1/VC (\%)), peak expiratory flow (PEF), operation parameters.

By comparing the postoperative respiratory parameters between the two groups and using independent samples $t$ test, it was found out that the difference in FVC between groups is 1.71 [95\% CI: -8.25 to 4.8 ] and it is not statistically significant $(t(155)=-0.52 ; p=0.60)$. Based on the Leuven test results FVC the distribution variance is not statistically notably different for group 1 and group $2(F=0.27, p=0.60)$.

Using routine and modified physiotherapy and comparing postoperative dynamic respiratory performance of the two groups, there was no statistically significant difference, proving that the two models are equally effective.
\end{abstract}

\section{Introduction}

During recent years the lifestyle of people has changed and the population is aging and cardiovascular diseases are an important problem, as evidenced by the high morbidity and hospitalization rates.

Various heart surgeries are one kind of the treatments of heart diseases. A variety of cardiac physiotherapy techniques are offered for reduction of complications during postoperative period in hospital. The most frequently mentioned techniques in literature and studies were chosen for this research - the promotion of expiration, coughing up, diaphragmatic breathing, the promotion of inhalation with inhalation spirometer [1-4]. 
Active movements of the limbs from the periphery to the centre, in lying, sitting, standing positions. Active movements of the body, strength stimulating techniques for major muscle groups, mm. gluteus max, mm. quadriceps, walking, climbing stairs. These techniques were applied in two different physiotherapy programmes - in programme 1 coughing up with holding the scar was done, diaphragmatic breathing, breath improvement, active movements of body and limbs from the periphery to the centre in lying, sitting, standing positions, walking and climbing stairs during seven post operation days. In the $2^{\text {nd }}$ programme - the promotion of inhalation with inspiratory spirometers, improvement of strength in the large muscle groups in lying, sitting, standing positions, walking and climbing stairs was carried out during seven postoperative days.

The most common complications in the postoperative period are atelectasis, infectious problems in the airways and the operation wound, pleural effusion, pain in surgery scar area and intercostal nerve localization sites, fatigue, dizziness, rhythm disorders. Physiotherapy programmes were developed in order to reduce postoperative complications [5-7].

The aim of the research is to study and compare the usability of two physiotherapy programmes, to analyze respiratory functions of patients before and after cardiac surgery in hospital during seven postoperative days (POD).

\section{Methods}

The research was carried out in Cardiac Surgery Centre of Pauls Stradins Clinical University Hospital from 2015 until the end of 2016. Ethical permissions from Riga Stradins University and Paul Stradins Clinical University Hospital Ethics Committees were obtained for carrying out the research. The participants of this study were informed about the progress of research and they have signed the informed consent form.

\subsection{Participants of study}

Patients with cardiovascular and valvular diseases, with a planned cardiac surgery aortocoronary bypass graft, valve replacement surgery and combined, median sternotomy, with artificial blood circulation, who move independently.

Exclusion criteria - acutely hospitalized patients, and patients who could not move independently. Participants were randomized into two groups, they were offered two distinct programmes under the guidance of a physiotherapist.

On the day before the planned heart surgery, the anthropometric data were defined pulse rate, blood pressure, respiratory rate, body mass index, weight, waist/hip circumference. Biochemical data - total cholesterol, high-density, low-density cholesterol, triglycerides, blood glucose levels. During spirography such dynamic indicators as forced vital capacity (FVC), forced expiratory volume in the $1^{\text {st }}$ second (FEV1), Tiffeneau index (FEV1/VC (\%)), peak expiratory flow (PEF) were defined, conclusions were made about the functionality of respiratory system. Spirographic data were redefined on the $6^{\text {th }}$ postoperative day.

On the seventh postoperative day a 6-minute walking test [8] was done, determining the distance travelled, heart failure functional class, pulse rate, blood pressure, oxygen saturation prior to the test, after the test, and after 5 minutes of recovery time. The patient's well-being was evaluated subjectively, using the Borg scale and Borg dyspnoea scale [9]. The test was carried out in 80-metre corridor, the corridor ends were marked which the participant went around, the walking took place as soon as it was possible, it was allowed to stop and rest during walking, if it was necessary, then to continue the test. The test could also be taken with the high walking frame, if it was necessary. 
The following data of heart surgery were analyzed: the duration of surgery, artificial blood circulation time, aortic occlusion time, body temperature, heart rate recovery mechanism.

\subsection{The evaluation of participants during the programme}

Before the exercises for every participant was measured pulse rate, blood pressure, oxygen saturation, the number of repetitions of exercises, the time of sleeping, seating, standing in minutes, the walking distance in metres, the number of climbed steps, the iteration number of breathing techniques, breath spirometer display in millilitres on each postoperative day, the duration of exercises in minutes. The exercises were carried out once a day, every day.

\subsection{Statistical analysis}

The results obtained were analyzed using descriptive statistics (mean values, standard deviation, percentage, minimum and maximum values), independent sample $t$ test and Leuven test.

\section{Results}

\subsection{Basic data}

The study (Table 1) included 157 participants who had undergone heart surgery via longitudinal sternotomy, valvular prosthesis 77 (49.04\%), bypass graft 57 (36.3\%), combined (valvular prosthesis and bypass graft) 23 (14.65\%). 72 (45.86\%) women and 85 $(54.14 \%)$ men participated in research. Participants were randomly split into two groups. Group 1 carried out a routine physical therapy programme, and the second group took a modified physiotherapy programme. There were $41(51.9 \%)$ women and $38(48.1 \%)$ men in the first group, the average age was $M=65.84(\mathrm{SD}=9.71)$. In this group there were 36 $(45.57 \%)$ valve replacement surgeries, $25(31.64 \%)$ grafts and $18(22.78 \%)$ combined surgeries. The average Body Mass Index (BMI) was $M=29.54(\mathrm{SD}=6.34)$, average respiratory rate before surgery was $M=20.18(\mathrm{SD}=3.96)$. Other tests, which were carried out before the operation, are illustrated in Table 1 . There were $31(39.74 \%)$ women and 47 (39.74\%) men in the second group. The average age was $M=67.26(\mathrm{SD}=8.73)$. In this group there were $31(39.73 \%)$ valve replacement, $32(41.02 \%)$ bypass and $15(19.23 \%)$ combined surgeries. The average BMI was $M=28.22(\mathrm{SD}=5.39)$ and average respiratory rate before surgery was $M=20.81(\mathrm{SD}=5.39)$. Both groups had 14 smokers.

\subsection{Data about surgeries}

The surgery was carried out in artificial blood circulation, one of the members from the first group, who had repeated surgery, was not tested and in the second group two patients, who had the operation without artificial blood circulation, were not tested. In the first group the artificial blood circulation time in minutes was $M=96.51(\mathrm{SD}=33.10)$, aortic clamping time in minutes was $M=64.96(\mathrm{SD}=22.10)$. The results are illustrated in Table 2 . In the second group artificial blood circulation time in minutes was $M=92.33(\mathrm{SD}=24.71)$, Aortic clamping time in minutes was $M=62.20(\mathrm{SD}=17.41)$. 
Table 1. Clinical characteristics data between first and second group participants $(n=157)$.

\begin{tabular}{|c|c|c|c|c|}
\hline & \multicolumn{2}{|c|}{ 1. group, $n=79$} & \multicolumn{2}{|c|}{ 2. group, $n=78$} \\
\hline & Mean & SD & Mean & SD \\
\hline Women:men & $41: 38$ & & $31: 47$ & \\
\hline Age, years & 65.84 & 9.71 & 67.26 & 8.73 \\
\hline Valve replacement surgery, $n$ & 36 & & 31 & \\
\hline Coronary artery bypass grafting, $n$ & 25 & & 32 & \\
\hline Combined heart surgery, $n$ & 18 & & 15 & \\
\hline Body mass index, $\mathrm{kg} / \mathrm{m}^{2}$ & 29.54 & 6.34 & 28.22 & 5.39 \\
\hline $\begin{array}{l}\text { Breathing frequency prior to } \\
\text { surgery, times per minute }\end{array}$ & 20.18 & 3.96 & 20.81 & 7.88 \\
\hline Smoking yes, $n$ :no, $n$ & $14: 65$ & & $14: 64$ & \\
\hline Total cholesterol, mmol/1 & 4.60 & 1.09 & 4.43 & 1.18 \\
\hline $\begin{array}{l}\text { Glucose, } \mathrm{mmol} / \mathrm{l} \\
6 \text { min walking test on } 7^{\text {th }}\end{array}$ & 6.34 & 2.11 & 5.86 & 1.53 \\
\hline postoperative day & 274.38 & 104.88 & 266.18 & 84.49 \\
\hline
\end{tabular}

Table 2. Description of intraoperative data for first and second group participants $(n=157)$.

\begin{tabular}{lcccc}
\hline & \multicolumn{2}{c}{ 1. Group, $n=78$} & \multicolumn{2}{c}{ 2. Group, $n=76$} \\
& Mean & SD & Mean & SD \\
\hline Cardiopulmonary bypass, min & 96.51 & 33.10 & 92.33 & 24.71 \\
Aortic clamping, min & 64.96 & 22.10 & 62.20 & 17.41 \\
Reperfusion, min & 23.49 & 10.08 & 23.40 & 9.53 \\
Lowest body temperature, ${ }^{\circ} \mathrm{C}$ & 35.85 & 0.39 & 35.78 & 0.54 \\
\hline
\end{tabular}

\subsection{Spirographic data}

Before and after surgery during spirography the dynamic breathing ratios were defined forced vital capacity (FVC), forced expiratory volume in the $1^{\text {st }}$ second (FEV1), Tiffeneau index (FEV1/VC (\%)), peak expiratory flow (PEF), maximal (mid-) expiratory flow (MEF 75, MEF 25). The results see in Table 3 . In the first group the evaluation of postoperative respiratory dynamic performance was not done for $2(2.53 \%)$ participants with complications - nosocomial infections (Acinetobacter baumannii) and flu, in the second group $2(2.56 \%)$ participants were not investigated, who had complications - neurological dysfunction un prolonged ICU ( 8 days). Repeated respiratory dynamic indicators were defined on the sixth postoperative day and they were compared between groups. The data were compared using independent sample $t$ test, it was concluded that the difference between group members of groups 1 and $2-\mathrm{FVC}$ difference is 1.71 [95\% CI: -8.25 to 4.8 ] and it is not a statistically significant difference $(t(155)=-0.52, p=0.60)$. Based on Leuven test results, it was concluded that FVC distribution variance is not statistically significantly different $(F=0.27 ; p=0.60)$ in the first and second groups. By analyzing other respiratory parameters with Leuven and $t$ tests, it was concluded that the result was similar. 
Table 3. Spirographic dynamical indicators for first and second group participants.

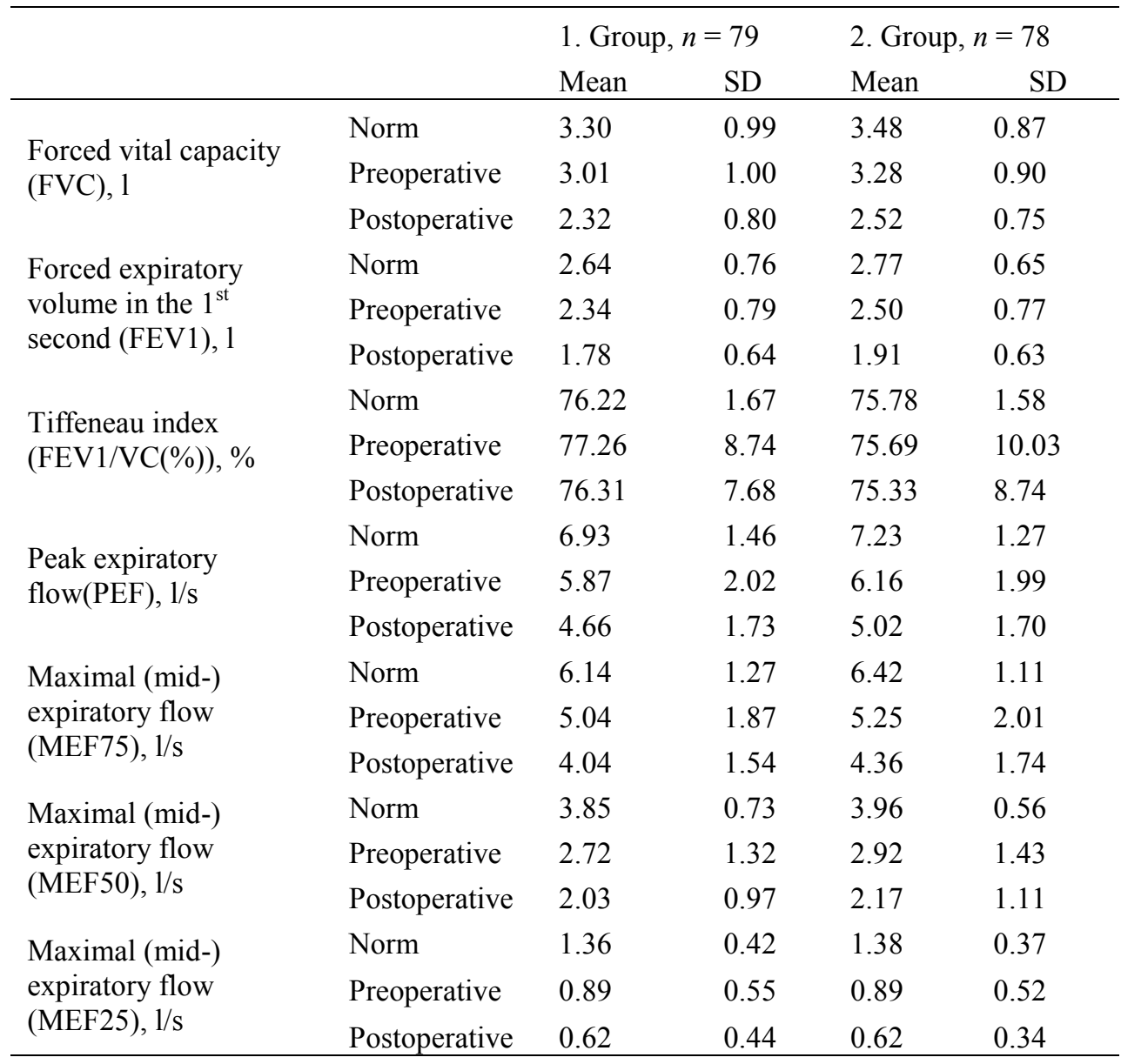

\subsection{Programmes}

During physiotherapy programmes for both groups of participants the vital parameters pulse, blood pressure, oxygen saturation was controlled. In Table 4 we see that physiotherapy programme starts on the first postoperative day (POD1), In the $1^{\text {st }}$ group 75 (94.9\%) participants start the programme and 4 participants do not start it, because of complications - 3 intubated, 1 with neurological dysfunction. 74 (94.87\%) participants from group 2 start the programme, 4 patients do not start, complications - 2 atrial fibrillation paroxysm, 2 had repeated operations. Physiotherapist guided exercises are held on seven POD. Participants are involved in the physiotherapy sessions from POD1 to POD4. From the $5^{\text {th }}$ POD the amount of activities decreases with starting independent activities of daily living.

Supervised coughing up technique was launched in two groups on the $1^{\text {st }}$ POD and it was continued till the $4^{\text {th }}$ POD for group 1 , but was reduced for group 2, which did inhalation spirometry technique. In the 2 nd group inhalation technique $(n=37)$ and muscle strength improvement $(n=28)$ starts on the $2^{\text {nd }}$ POD and is intensively used till the $5^{\text {th }}$ POD. Inhalation promotion technique $(n=24)$ for the $1^{\text {st }}$ group starts on the $2^{\text {nd }}$ POD and continues till $5^{\text {th }}$ POD. The time of activities in minutes is growing up till the $4^{\text {th }} \mathrm{POD}$, then 
remains constant. The intensity of activities (meters walked and the amount of climbed steps) is rising till the $6^{\text {th }}$ POD for both groups.

\subsection{6-minute walk test}

On the $7^{\text {th }}$ postoperative day the members of groups 1 and 2 carried out a 6 -minute walking test to compare the distance walked. Two participants from group 1 did not take part in 6minute walk test, one refused, the other had nosocomial infection (Acinetobacter baumannii). One patient from group 2 died. For the $1^{\text {st }}$ group the walked distance in metres was $M=274.38(\mathrm{SD}=104.88)$. For the $2^{\text {nd }}$ group the walked distance in metres was 266.18 $(\mathrm{SD}=84.49)$. Using the independent sample $t$ test, it was concluded that the difference of the distance in metres of 6-minute walking test for groups 1 and 2, was 11.86 meters [95\% CI: -16.23 to 39.95$]$ and has no statistically significant difference $(t(152)=0.83 ; p=0.15)$. On the basis of results of Leuven test a conclusion was made that for groups 1 and 2 walking distance variance does not have significant difference $(F=1.82, p=0.18)$.

\section{Discussion}

The aim of cardiac rehabilitation is to return the patients' daily activities and social environment as quickly as possible, at the same time preserving human functioning in high physical and psychological level. The study used two different physiotherapy programmes. For the first programme expiration stimulating techniques were applied, the second programme used inhalation stimulating techniques and strength in the large muscle groups was improved. In both groups early mobilization was applied. Inhalation spirometry and the improvement of inspiratory muscle strength is one of the methods described in many studies [10-13]. It has been proved that this method is effective, but there are more effective programmes, that offer variety of techniques such as expiratory promoting, early mobilization, aerobic exercises [10-15]. The study with healthy people demonstrated that inhalation spirometry training shows the best results [16]. The aim of research was to find out which of the applications of this technique are more effective. Basic data for both groups as age, breathing frequency before the operation, the number of patients who smoke were similar. Spirographic results before the operation for two groups of patients were similar. Duration of artificial blood circulation during surgery for patients from group 1 was 96.51 minutes, for patients from group 2 it was 92.33 minutes which is not significantly different. On the sixth postoperative day spirography was repeatedly carried out, concluding that results in both groups are not significantly different. On the seventh postoperative day a 6-minute walk test was done to check the functional indicators for both groups. 6-minute walk test results did not differ significantly between the two groups, Group 1 on average walked 274.38 metres, the second group walked on average 266.18 metres. 6-metre walking test is recommended as a good tool for people with moderate disabilities $[8,17]$. Different physiotherapy methods applied for the first and second groups show similar results for both - assessing spirographic data and analysis of the 6-meter walking test results after surgery. Both applied physiotherapy programmes were equivalent and applicable to patients in the period after cardiac surgery in hospital. Different physiotherapy techniques applied in programmes could be combined and successfully applied for faster return of patients to daily activities and social environments. Physiotherapy programs after heart operations should be standardized, they can be carried 


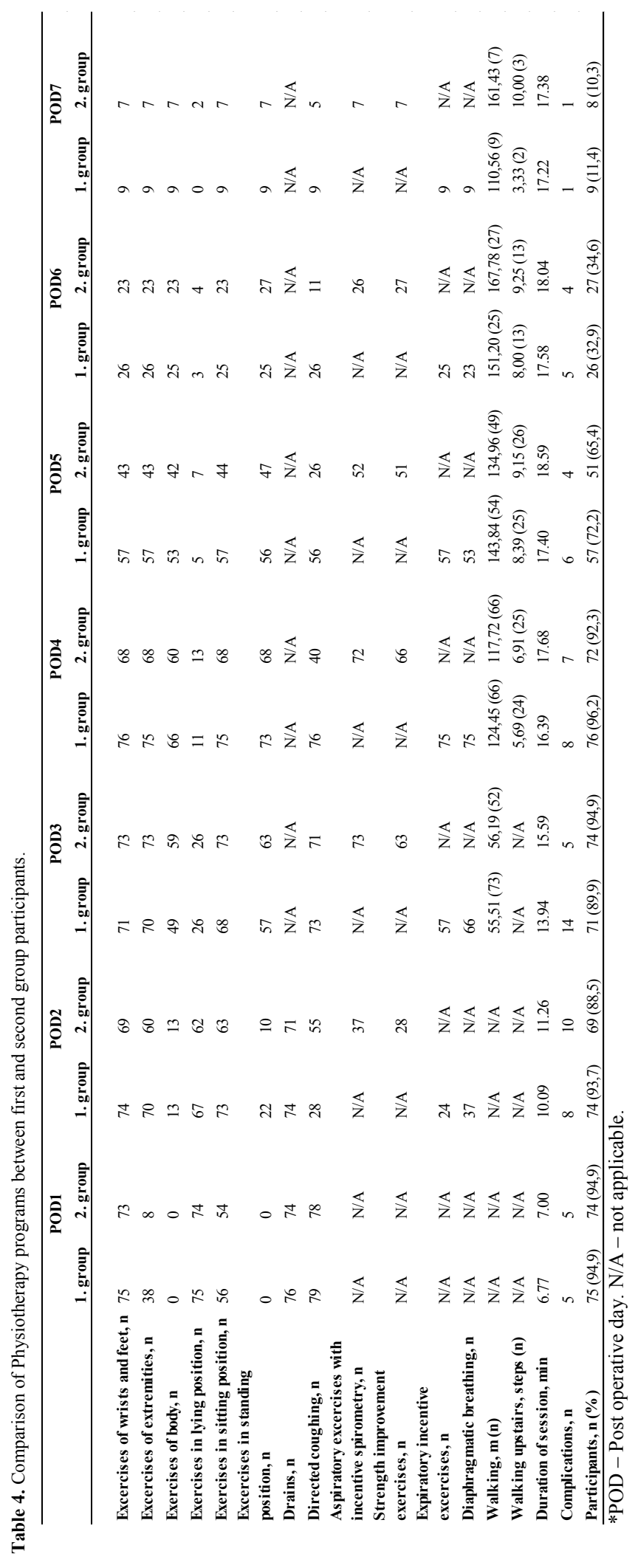


out only on weekdays, or on all days of the week $[5,18]$. Screening techniques are being searched that would anticipate the length of stay in hospital and a 5-metre walking test has been offered [19]. Good results and reduced complications are demonstrated by programs that started before the operation and continue in the postoperative period [20,21]. Similar to our study, the highest number of complications is on the first three POD, the most common complications - respiratory, hemodynamic and cardiac conduction disorders $[5,6$, 22]. In our study the patients performed independent physical activities from the $4^{\text {th }}$ POD. If twenty years ago it was considered as unethical model [22], then currently secure, independent activity models are being studied under telemetry monitoring [23].

\section{Conclusion}

In this study, it is concluded that a variety of physiotherapy techniques for improvement of respiratory functions, as well as the preservation and improvement of mobility, and the promotion of strength during postoperative period are equivalent and can be included in the total, standardized model of physiotherapy for reducing complications after cardiac surgery in hospital period.

\section{References}

1. R.D. Restrepo, R. Wettstein, L. Wittnebel, M. Tracy, Incentive Spirometry: 2011 Clinical Practice Guideline, Respiratory Care 56, 1600-1604 (2011)

2. C.M. Mans, J.C. Reeve, M.R. Elkins, Postoperative outcomes following preoperative inspiratory muscle training in patients undergoing cardiothoracic or upper abdominal surgery : a systematic review and meta analysis, Clinical Rehabilitation 29(5), 426-438 (2015)

3. V.K. Ravaliya, Functional Status in Post-CABG Patients Following Physiotherapy A Retrospective Analysis, Indian Journal of Physiotherapy and Occupational Therapy 7(3), 270-274 (2013)

4. E. Crisafulli, E. Venturelli, G. Siscaro, Respiratory muscle training in patients recovering recent open cardiothoracic surgery: a randomized controlled trial, BioMed Research International, Article ID 354276, 1-7 (2013)

5. E.R. Winkelmann, F. Dallazen, A.B.S. Bronzatti, I.C.W. Lorenzoni, P. Windmöller, Analysis of steps adapted protocol in cardiac rehabilitation in the hospital phase, Brazil J Cardiovasc Surg 30, 40-48 (2015)

6. V.O. Carvalho, Phase 1 cardiovascular rehabilitation: be aggressive?, Journal of Cardiothoracic Surgery 6, 140 (2011)

7. R.M. Macedo, J.R. Faria-Neto, C. Constantini, Phase I of cardiac rehabilitation: a new challenge for evidence based physiotherapy, WJ of cardiology 3(7), 248-255 (2011)

8. Approved by the ATS Board of Directors, ATS Statement: Guidelines for the SixMinute Walk Test, Am J Respir Crit Care Med 166, 111-117 (2002)

9. Borg GA, Psychophysical bases of perceived exertion, Med Sci Sports Exerc, 14, 377-381 (1982)

10. S. Buurena, E.H.J. Hulzebosc, K. Valkenetd, R. Lindemand, Reference chart of inspiratory muscle strenght: a new tool to monitor the effect of pre-operative training, Physiotherapy 100(2), 128-133 (2014)

11. A.L. Narayanan, J. Bahru, S.R. Hamid, E. Supriyanto, Evidence regarding patient compliance with incentive spirometry interventions after cardiac, thoracic and abdominal surgeries: A systematic literature review, CJRT 52(1), 17-26 (2016) 
12. P. Palau, E. Dominguez, E. Nunez, Effects of inspiratory muscle training in patients with heart failure with preserved ejection fraction, EJ of Preventive Cardiology 21(12), 1465-1473 (2014)

13. C.R. Carvalho, D. Paisani, A. Lunardi, Incentive spirometry in major surgeries: a systematic review, Brazilian Journal of Physical Therapy 15(5), 343-50 (2011)

14. H.S. García, L.E. Prendes, J.M. Oquendo, A. Jose, E.R. Estany, L.E. Prendes, Hospitalization phase of cardiac rehabilitation. Protocol for cardiac surgery, CorSalud 6(3), 246-256 (2014)

15. D. Karaszewski, Comparison of two models of hospital rehabilitation in patients after coronary artery bypass grafting, Termedia Country of Publication 11, 86-95 (2015)

16. D.N. Paiva, L.B. Lindstrand, D.F. Bordin, R. Gass, Revista Portugesa de pneumonia, Inspiratory muscle

17. D.L. Borges, M.G. Silva, Silva, L. Nascimento, J.V. Fortes, E.T. Costa, R.P. Assunção, C.M. Lima, N.V.J. da Silva, M. Bernardo-Filho, D.S. Caputo, Effects of Aerobic Exercise Applied Early After Coronary Artery Bypass Grafting on Pulmonary Function, Respiratory Muscle Strength, and Functional Capacity: A Randomized Controlled Trial, Index Medicus 13(9), 946-951 (2016)

18. E. Westerdahl, M. Möller, Physiotherapy - supervised mobilization and exercise following cardiac surgery: a national questionnaire survey in Sweden, Journal of Cardiothoracic Surgery 5, 67 (2010)

19. C.M. Wilson, S.R. Kostsuca, S.M. McPherson, M.C. Warren, J.W. Seidell, R. Colombo, Preoperative 5 - Meter Walk Test as a Predictor of Length of Stay After Open Heart Surgery, Cardiopulmonary Physical Therapy Journal 26(1), 2-7 (2015)

20. A. Herdy, Pre and postoperative cardiopulmonary rehabilitation in hospitalized patients undergoing coronary artery bypass surgery, American journal of Physical Medicine \& Rehabilitation 87(9), 714-719 (2008)

21. C. Urell, E. Westerdahl, H. Hendenstrom, Lung function before and two days after open heart surgery, Critical Care Research and Practice, Article ID 291628, 1-7 (2012)

22. D. Johnson, C. Kelm, D. Thomson, B. Burbridge, I. Mayers, The Effect of Physical Therapy on Respiratory Complications Following Cardiac Valve Surgery, Chest 109, 638-44 (1996)

23. S.F. Mungovan, P. Singh, G.C. Gass, N.A. Smart, A.D. Hirschhorn, Effect of physical activity in the first days after cardiac surgery, J Rhabil Med 49, 71-77 (2017) 
